\title{
MATHEMATICAL TOPICS IN NONLINEAR KINETIC THEORY II
}


This page is intentionally left blank 
Series on Advances in Mathematics for Applied Sciences - Vol. 1

\title{
MATHEMATICAL TOPICS IN NONLINEAR KINETIC THEORY II
}

\author{
THE ENSKOG EQUATION
}

\section{N. Bellomo}

Politecnico di Torino, Italy

M. Lachowicz

University of Warsaw, Poland

J. Polewczak

State University of New York at Stony Brook, USA

G. Toscani

University of Ferrara, Italy 


\author{
Published by \\ World Scientific Publishing Co. Pre. Lud. \\ P O Box 128, Farrer Road, Singapore 9128 \\ USA office: 687 Hartwell Street, Teeneck, NJ 07666 \\ UK office: 73 Lynton Mead, Totteridge, London N20 8DH
}

\title{
MATHEMATICAL TOPICS IN NONLINEAR KINETIC THEORY II:
}

THE ENSKOG DQUATION

Copyright @ 1991 by Word Scientific Publishing Co. Pte. Ltd.

All rights reserved. This book, or parts thereof, may not be repraduced in any form or by any means, electronic or mechanical, including photocopying, recording or any information storage and retrieval system now known or to be invented, without written permission from the Publisher.

ISBN $981-02-0447-7$

Printed in Singapore by JBW Printers \& Binders Pte. Ltd. 


\section{ACKNOWLEDGMENTS}

N. B. and G. T. acknowledge the partial support both of the Italian Minister for University, Scientific and Technological Reserch M. U. R. S. T. and of the National Research Council, C. N. R. (Gruppo Nazionale Fisica Matematica).

M. L. acknowledges the partial support of the Minister of National Education of Poland under Grant no. RP. I. 10.

J. P. acknowledges the support of National Science Foundation Grant no. CHE881490. 
This page is intentionally left blank 


\section{PREFACE}

As known, when one deals with a rarefied gas, namely a gas with a mean free path larger than the characteristic dimensions of the particles, the equations of continuum mechanics (i.e. Euler and Navier-Stokes), cannot be used any longer to describe the behaviour of the physical system, but they have to be replaced by the equations of the kinetic theory of gases.

Certainly the celebrated Boltzmann equation is the most interesting and successful model for a simple dilute monoatomic gas. This success has been surrounded by several controversies and criticisms essentially focused on its derivation based upon the hypothesis of molecular chaos, which, as it seems, might not have the support of a mathematical proof.

Nevertheless several interesting and relevant problems in gas-dynamics have been solved by the application of the Boltzmann equation. Moreover, the mathematical problem of the analysis of existence and uniqueness of the solutions to the initial and initial-boundary value problem has now several interesting results obtained after several unsuccessful and a few successful attempts by applied mathematicians.

The reader will find in the book [1] all pertinent literature which appeared before its edition in 1988, namely the literature on existence and uniqueness theorems for the solution to the initial value problem in the case of small perturbation of equilibrium or vacuum. In addition to these results, one has to acknowledge the fundamental 
viii THE ENSKOG EQUATION

paper by DiPerna and Lions [2], appeared after the edition of [1], which has shown that for a gas with finite mass and energy the initial value problem always has a solution (even if uniqueness is lost) also in the case of large initial data.

Several important problems still remail open: uniqueness for large $L_{1}$-data, existence of solutions for a gas with infinite mass or existence of shock profiles and so on. However, it is plain that relevant progresses in this field have been realized, as documented in [1] and [2], in the last few years.

However, the validity of the Boltzmann equation can be accepted only for a rarefied gas. On the other hand mathematical models are needed to describe the physical system in the range from the rarefied to the continuum flow.

An interesting and certainly successful attempt to take into account, at least some of the various dense gas effects, is the one due to Enskog [3], who proposed a model which, still remaining in the scheme of binary collisions, takes into account the influence of the overall dimensions of the spheres, whereas the Boltzmann-like description is the one of point masses.

Enskog model, derived under somewhat heuristic arguments, has been afterwards revised by several Authors (in particular by Resibois [4]) who proposed some revised models (or class of models) consistent with irreversible thermodynamics.

The Enskog equation has certainly found the favour of physicists. In fact it is considered an interesting and valid substitute to the Boltzmann equation which allows, among the other things, a more accurate computation of the transport coefficients [5]. Nevertheless the literature on the mathematical aspects of this equation is somewhat limited, being essentially originated by papers [6] and [7] and then developed through various papers all cited in the book [1]. 
PREFACE ix

Also in the case of the Enskog equation several interesting mathematical results are now available in the literature even if the problem of its derivation has, as for the Boltzmann equation, several obscure aspects which are still waiting for some enlightening.

This book has the purpose of providing the state-of-the-art on the analysis of the mathematical aspects of the initial and initial-boundary value problem. This in view both of further developments of the mathematical analysis and of the modelling of dense fluids in the framework of kinetic theory.

The plan of the book is presented and discussed at the end of the first chapter, which also provides a description and a classification of the Enskog-type models. Even though this chapter points out some advantages of this model, it does not hide the criticisms which may be addressed to the model itself. One of the purposes of this book is, however, to provide an accurate mathematical analysis of the model in the convinction that this may contribute to the improvement of the model itself. All this having in mind that the final goal of mathematical modelling is to provide a model suitable to describe flow fields in physical regimes spanning from the continuum to the rarefied flow.

This book is in five chapters. The second chapter after the introduction of Chapter 1, deals with the initial value problem for initial data closed to vacuum. This chapter also analyses an $H$-theorem for the Enskog equation when an existence and uniqueness theorem holds for the solution to the initial value problem. Chapter 3 deals with the initial value problem for large initial $L_{1}$ data. The analysis is developed in the framework of the already mentioned theorem by DiPerna and Lions which is furtherly developed in order to deal with Enskog-type models. Chapter 4 deals with the so called asymptotic theory for the Enskog equation consisting in the analysis 
of the behaviour of the solutions to mathematical problems when the radius of the spheres and the Knudsen number tend to zero: namely on the trend of the Enskog equation to the Boltzmann equation and on the hydrodynamic limit. The final chapter provides a discussion on several open problems and some suggestions in the direction of new research lines.

The Authors wish to express their gratitude to William Greenberg (Virginia Tech), Andrzej Palczewski (Warsaw University), Luigi Preziosi (Politecnico di Torino), George Stell (SUNY at Stony Brook) for helpful discussions during the preparation and revision of the manuscript.

\section{References}

[1] N. Bellomo, A. Palczewski and G. Toscani, Mathematical Topics in Nonlinear Kinetic Theory, World Scientific, (1988).

[2] R. DiPerna and P. L. Lions, Comp. Rend. Acad. Sci. Paris, 306, 851-859, (1988).

[3] D. Enskog, Svenska Akad., 63, (1921).

[4] P. Resibois, J. Statist. Phys.,19, 593-609, (1978).

[5] P. Resibois and M. De Leener, Classical Kinetic Theory of Fluids, Wiley, (1977).

[6] M. Lachowicz, Bull. Polish Acad. Sci., 31, 89-93, (1983).

[7] G. Toscani and N. Bellomo, in Advances in Hyperbolic Equations, Spec. Issue in Comp. Math. Appl., 13, 851-859, (1987). 


\section{CONTENTS}

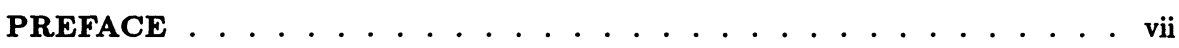

\section{Chapter 1: THE NONLINEAR ENSKOG EQUATION}

1.1 The One Particle Distribution Function and the Boltzmann

Equation. . . . . . . . . . . . . . 1

1.2 The Standard Enskog Equation _. . . . . . . . . . 9

1.2 .1 Method 1 . . . . . . . . . . . . . . 12

1.2 .2 Method 2 . . . . . . . . . . . . . . . . . 14

1.3 The Revised Enskog Equation . . . . . . . . . . . . . 18

1.4 Classification of Enskog Type Models . . . . . . . . . . . 23

1.5 On Mathematical Formulation of Problems . . . . . . . . 25

1.6 Plan of the Book . . . . . . . . . . . . . 27

References. . . . . . . . . . . . . . 31

Chapter 2: THE INITIAL VALUE PROBLEM FOR DECAYING DATA

2.1 Introduction . . . . . . . . . . . . 35

2.2 Existence and Uniqueness: Basic Notations . . . . . . . . 38

2.3 Existence and Uniqueness of Mild Solutions . . . . . . . . . 43

2.4 Existence and Uniqueness of Classical Solutions. . . . . . . . 56

2.5 Asymptotic Behaviour in Time . . . . . . . . . . . . 65

2.6 On the $H$-Theorem for the Abstract(Revised) Enskog Equation . . . 72

References. . . . . . . . . . . . 81

Chapter 3: THE CAUCHY PROBLEM FOR LARGE $\mathrm{L}_{\mathbf{1}}$ DATA

3.1 Introduction . . . . . . . . . . . . . 85

3.2 Basic a Priori Estimations . . . . . . . . . . . . . . 86

3.3 Formulation of Existence Theorems . . . . . . . . . . 101 
3.4 Convergence and Stability Results . . . . . . . . . . . 108

3.5 Existencce and Some Properties of Approximate Solutions . . . . 126

References. . . . . . . . . . . . . . . . 145

\section{Chapter 4: ON THE ASYMPTOTIC THEORY FOR THE ABSTRACT ENSKOG EQUATION}

4.1 Introduction . . . . . . . . . . . . . . . . . . . . 149

4.2 Asymptotic Equivalence between the Abstract Enskog Equation and the Boltzmann Equation for Decaying Data . . . . . . . . 154

4.3 Asymptotic Equivalence between the Abstract Enskog

Equation and the Boltzmann Equation in $L_{1}$. . . . . . . . . . 166

4.4 Some Open Problems . . . . . . . . . . . . . . . . . . . . 167

4.4.1 Asymptotic Behaviour of Solutions of the Abstract Enskog Equation for Small Knudsen Numbers . . . . . . . . . 168

4.4.2 Asymptotic Equivalence between the Abstract Enskog

Equation and the Boltzmann Equation in the Presence of an External Force Field. . . . . . . . . . . . . . . 176

References. . . . . . . . . . . . . . . . . . 181

\section{Chapter 5: OPEN PROBLEMS ON THE MATHEMATICAL THEORY OF THE ENSKOG EQUATION}

5.1 Introduction . . . . . . . . . . . . . . . . . 185

5.2 On the Initial-Boundary Value Problem . . . . . . . . . . . . 186

5.3 On the Initial Value Problem . . . . . . . . . . . . . . . 190

5.3.1 On the Initial Value Problem for Decaying Data . . . . . . 191

5.3.2 Uniqueness in the Initial Value Problem with Large

$L_{1}$ Initial Conditions . . . . . . . . . . . . . . . 192

5.3.3 Analysis of Some Aspects of Fluid-Dynamic Flows . . . . . 192

5.4 On the Mathematical Modelling . . . . . . . . . . . . . . 194

5.5 On the Semidiscrete Enskog Equation . . . . . . . . . . . . . 197

References. . . . . . . . . . . . . . . . 200

AUTHOR INDEX . . . . . . . . . . . . . . . 203 